\title{
Sparsity enforcing edge detection method for blurred and noisy Fourier data
}

\author{
W. Stefan; A. Viswanathan ${ }^{\dagger}$ A. Gelb \\ Rev : 9 August 29, 2011
}

\begin{abstract}
We present a new method for estimating the edges in a piecewise smooth function from blurred and noisy Fourier data. The proposed method is constructed by combining the so called concentration factor edge detection method, which uses a finite number of Fourier coefficients to approximate the jump function of a piecewise smooth function, with compressed sensing ideas. Due to the global nature of the concentration factor method, Gibbs oscillations feature prominently near the jump discontinuities. This can cause the misidentification of edges when simple thresholding techniques are used. In fact, the true jump function is sparse, i.e. zero almost everywhere with non-zero values only at the edge locations. Hence we adopt an idea from compressed sensing and propose a method that uses a regularized deconvolution to remove the artifacts. Our new method is fast, in the sense that it only needs the solution of a single $l_{1}$ minimization. Numerical examples demonstrate the accuracy and robustness of the method in the presence of noise and blur.
\end{abstract}

\section{Introduction}

We are concerned with the accurate and robust detection of jump discontinuities (edges) in piecewise smooth functions where only a finite number of Fourier coefficients are available. Such information can be used to improve the reconstructions, restorations and classifications of signals. For instance, knowing the edge locations, or more precisely the intervals of smoothness, is necessary for high order reconstruction of a signal from a truncated Fourier series, see e.g. [12, 21]. As another example, [22] uses edge information to eliminate the stair case effect typical for total variation (TV) restorations and reconstructions. Finally, in [15] edge data is used to decrease the number of samples needed for reconstructions from partial Fourier data.

The so called concentration factor (CF) method, proposed in $[9,10]$, directly uses the given Fourier data to detect the location of edges. A good overview can be found in [25]. The method approximates the corresponding jump function ${ }^{1}$ of a piecewise smooth function by converging to its singular support. One advantage of the CF method is that it can be made to be high order,

\footnotetext{
*Department of Imaging Physics, The University of Texas, MD Anderson, wstefan@mdanderson.org

${ }^{\dagger}$ Department of Computing and Mathematical Sciences, California Institute of Technology, aditya.v@caltech.edu

${ }^{\ddagger}$ School of Mathematical \& Statistical Sciences, Arizona State University, anne.gelb@asu.edu

${ }^{\S}$ School of Mathematical \& Statistical Sciences, Arizona State University, renaut@asu.edu

${ }^{1}$ The jump function is a function that is zero away from jump locations (edges) and takes on the value of the jump at the jump locations
} 
in the sense that it converges quickly to zero away from jump locations when certain high order concentration factors are chosen. However, since the method is global, it suffers from the Gibbs phenomenon. Specifically, when high order CFs are used, the resulting ringing artifacts near the jump discontinuities can lead to the misidentification of edges when simple thresholding is used to differentiate the edges from smooth regions. (Low order CFs may not properly resolve the function variation in smooth regions, causing the misclassification of edges there.) Nonlinear filtering is able to remove or suppress some of the oscillations, $[11,25,8,6]$. Unfortunately these techniques become less effective when the Fourier data is at all corrupted (e.g. by noise or blurring), or when some of the data is missing. In particular, additional ringing artifacts and aliasing affect the results.

In [26], the authors describe three extensions of the CF method to combat the effects of partial and/or noisy Fourier data. One extension "fills" in missing Fourier coefficients and suppresses oscillations by minimizing the TV semi-norm. Here we expand on this minimization technique, but propose an alternative to the TV semi-norm in order to exploit the structure of the oscillations generated by the original CF method. Our new method can be applied when only partial Fourier coefficients are available as well as when the given data is contaminated by blurring or noise. The proposed method approximates the associated jump function of a piecewise smooth function and removes the Gibbs oscillations and aliasing effects that are introduced by the CF method by performing a deconvolution with its so called matching waveform, [8], and imposing sparseness on the jump function approximation. The jump function approximation is found in a single $l_{1}$ minimization step. As with the compressive sensing (CS) edge detection method in [26], we combine ideas from CS with the CF method [9, 10]. However we do not suppress the oscillations introduced by the CFs by minimization of the TV semi-norm, but rather remove them by a regularized deconvolution step. We also use the concept of the matching waveform introduced in [8] to estimate the point spread function (PSF) of the oscillations and solve a sparse deconvolution problem. The method can be applied to any CF that satisfies an admissibility condition and provides sparse and robust results for the test cases we have considered. Finally, our method naturally handles data that contains blurring because it is based on Fourier data rather than data in physical space.

We solve the edge detection problem for a one-dimensional test function and consider four different cases:

Case 1: Clean Fourier Data: This is the best case scenario in which acquired Fourier data is assumed to be uncorrupted by undersampling (case 2), blur (case 3) or noise (case 4). Note that since we are only considering a finite number of Fourier coefficients, the problem is still non-trivial due to truncation error and Gibbs phenomenon.

Case 2: Undersampling: Here we assume that only partial Fourier data is available. Fourier coefficients are deleted from the middle of the spectrum (symmetrically), i.e. both low as well as high frequencies are still present. We vary the size of the bandwidth of missing Fourier data. In the context of minimization problems, the missing band of Fourier data corresponds to the classic case of undersampling.

Case 3: Blur: We assume that the data is blurred by application of a spatially invariant blur applied directly to the Fourier coefficients. In this case the edges in the signal are "smoothed out" making edge detection using classical methods very difficult.

Case 4: Noise: We explore the impact of additive independent and identically distributed (i.i.d.) complex Gaussian noise in the Fourier coefficients, which is also in general a challenge for 
edge detection by classical methods.

Our numerical results for these four cases demonstrate the robustness of our proposed method with respect to how the regularization parameter for the minimization process is chosen. The method correctly identifies edges in the test signal for a large range of regularization parameters. Not surprisingly, how that range is chosen depends on the quality of Fourier coefficients with respect to noise and blurring, as well as the amount of missing Fourier data.

Finally, as an extension of these four cases, we also consider the situation where the given Fourier data is nonuniform. Non-Cartesian Fourier data is sometimes collected in applications such as magnetic resonance imaging (MRI), [1]. While the standard CF method may fail in this case, [28], our proposed method is able to capture the edges in a one-dimensional piecewise smooth function given nonuniform Fourier data.

The paper is organized as follows: In Section 2 we give a brief review of the results in $[9,10]$ that are relevant for this paper. In Section 3 we combine CS with the CF method to remove the oscillations from the jump function approximation. Numerical results demonstrate the success of the method when applied to each of the four cases. Section 4 discusses the recovery of edges from non-harmonic spectral data. The challenge posed by non-harmonic acquisitions is briefly described, along with a variant of the formulation in Section 3 applicable to non-harmonic spectral data. Section 5 provides some concluding remarks and ideas for future investigations.

\section{The concentration factor edge detection method}

\subsection{Background}

Let

$$
\hat{f}_{k}=\frac{1}{2 \pi} \int_{-\pi}^{\pi} f(x) e^{-i k x} d x, \quad k=-N, \cdots, N
$$

be the first $2 N+1$ Fourier coefficients of a piecewise continuous $[-\pi, \pi]$ periodic function, $f$, where $i=\sqrt{-1}$. We define the corresponding jump function as

$$
[f](x)=f\left(x^{+}\right)-f\left(x^{-}\right) .
$$

Note that the jump function is zero everywhere except at the jump discontinuities where it takes on the corresponding jump value. Our goal is to approximate (2) assuming that we are given the first $2 N+1$ blurred, noise-corrupted Fourier coefficients,

$$
\hat{g}_{k}=\hat{f}_{k} \hat{h}_{k}+\hat{n}_{k}, \quad k=-N, \cdots, N
$$

where $\hat{h}_{k}$ are Fourier coefficients of a known point spread function and $\left\{\hat{n}_{k}\right\}_{k=-N}^{N}$ is i.i.d. complex Gaussian noise. We first consider edge detection for a function that is free of noise and blur (case 1).

The concentration factor $(\mathrm{CF})$ edge detection method, $[9,10]$, approximates the jump function, $(2)$, as

$$
S_{N}^{\sigma}[f](x)=i \sum_{k=-N}^{N} \sigma\left(\frac{|k|}{N}\right) \operatorname{sgn}(k) \hat{f}_{k} e^{i k x}=\left(f * C_{N}^{\sigma}(x)\right), \quad C_{N}^{\sigma}(x):=\sum_{k=1}^{N} \sigma\left(\frac{k}{N}\right) \sin (k x) .
$$


The convergence properties of (4) depend on the choice of the concentration factor used, $\sigma(\eta)=$ $\sigma\left(\frac{|k|}{N}\right)$, which must satisfy the admissibility conditions (see [10] for more details):

1. $C_{N}^{\sigma}(x)$ is odd

2. $\frac{\sigma(\eta)}{\eta} \in C^{2}(0,1)$

3. $\int_{\epsilon}^{1} \frac{\sigma(\eta)}{\eta} \rightarrow-\pi, \epsilon=\epsilon(N)>0$ is small

These admissibility conditions require that $C_{N}^{\sigma}$ is odd and suitably normalized in order to approximate $[f](x)$, and that $\sigma(\eta)$ have some regularity.

If $d(x)$ denotes the distance between a point in the domain and the nearest discontinuity, then (4) converges to $[f](x)$ as

$$
S_{N}^{\sigma}[f](x)=[f](x)+\left\{\begin{aligned}
\mathcal{O}\left(\frac{\log N}{N}\right) & d(x) \leq \frac{\log N}{N} \\
\mathcal{O}\left(\frac{\log N}{(N d(x))^{s}}\right) & d(x)>>\frac{1}{N}
\end{aligned}\right.
$$

The convergence rate $s>0$ depends upon the concentration factor chosen $[10,6]$.

Two examples of CFs derived in $[9,10]$ include

$$
\begin{aligned}
\sigma_{\text {poly }}(\eta) & =\pi p \eta^{p} \\
\sigma_{\exp }(\eta) & =\pi \gamma \eta \exp \left(\frac{1}{\alpha \eta(\eta-1)}\right) \quad \text { where } \quad \gamma=\int_{1 / N}^{1-1 / N} \exp \left(\frac{1}{\alpha \rho(\rho-1)}\right) d \rho
\end{aligned}
$$

where $p \in \mathbb{N}^{+}$and $\alpha>0$ denote the order of the CF. While there are other admissible CFs, this investigation will focus on the difference between higher and lower order methods, in particular between a lower order polynomial CF with $p=1$, a higher order polynomial CF with $p=2$, and the higher order exponential CF. Figure 1 illustrates the two polynomial CFs $\sigma_{1}(\eta)=\eta$, and $\sigma_{2}(\eta)=2 \eta^{2}$, and the exponential $\mathrm{CF} \sigma_{\exp }(\eta)$. Notice that the polynomial CFs only filter out low frequencies, while the exponential CF also filters out high frequency components. We emphasize that high order refers to the convergence to zero away from the jump discontinuities, since $\mathcal{O}(1)$ oscillations form as the jump discontinuities of $[f](x)$ are approached, and is expected since we are using a high order global method to approximate piecewise smooth $[f](x)$. We illustrate this with the following example:

$$
f(x)=\left\{\begin{array}{rll}
3 / 2 & \text { for } & -\frac{3 \pi}{4} \leq x<-\frac{\pi}{2} \\
7 / 4-x / 2+\sin (7 x-1 / 4) & \text { for } & -\frac{\pi}{4} \leq x<\frac{\pi}{8} \\
x 11 / 4-5 & \text { for } & \frac{3 \pi}{8} \leq x<\frac{3 \pi}{4} \\
0 & & \text { otherwise. }
\end{array}\right.
$$

Figure 2 shows the CF method jump function approximation to (7) using different CFs and demonstrates that the price to be paid for fast convergence to zero away from a jump discontinuity is more oscillations around the jump.

As shown in [11], one way of mitigating the oscillations is to apply the minmod function,

$$
\operatorname{minmod}\left\{a_{1}, a_{2}, \ldots, a_{n}\right\}:= \begin{cases}s \min \left(\left|a_{1}\right|,\left|a_{2}\right|, \ldots,\left|a_{n}\right|\right) & \text { if } \operatorname{sgn}\left(a_{1}\right)=\cdots=\operatorname{sgn}\left(a_{n}\right):=s \\ 0 & \text { otherwise }\end{cases}
$$




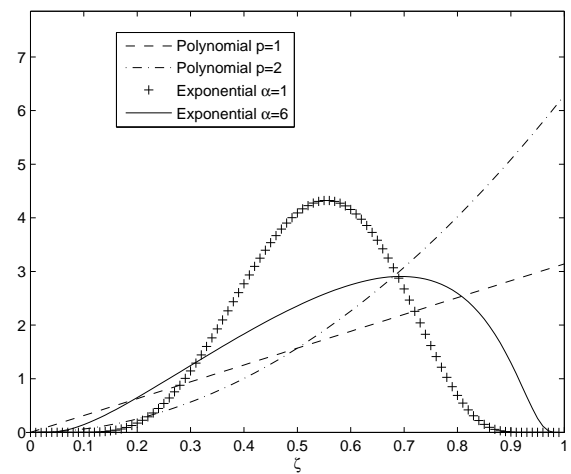

Figure 1: Illustration of the CFs $\sigma_{1}$ (dash), $\sigma_{2}$ (dash dot) and $\sigma_{\exp }$ (pluses for $\alpha=1$ and solid line for $\alpha=6)$. Here and throughout the paper, in the definition for $\sigma_{\exp }, \alpha=1$ is chosen.

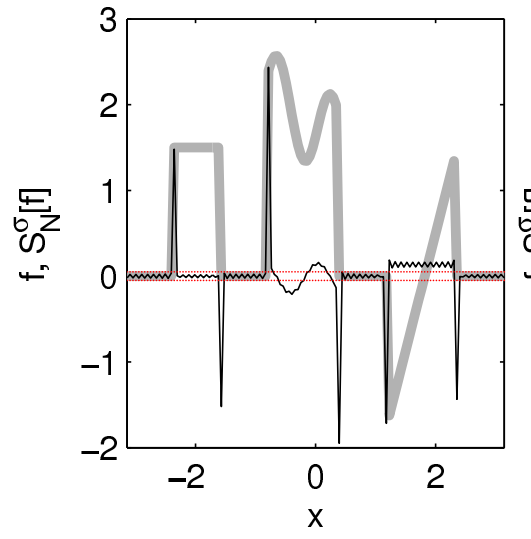

(a) Polynomial $p=1\left(\sigma_{1}\right)$

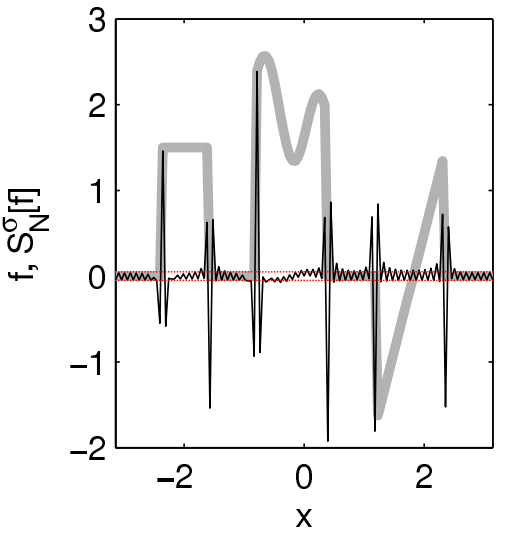

(b) Polynomial $p=2\left(\sigma_{2}\right)$

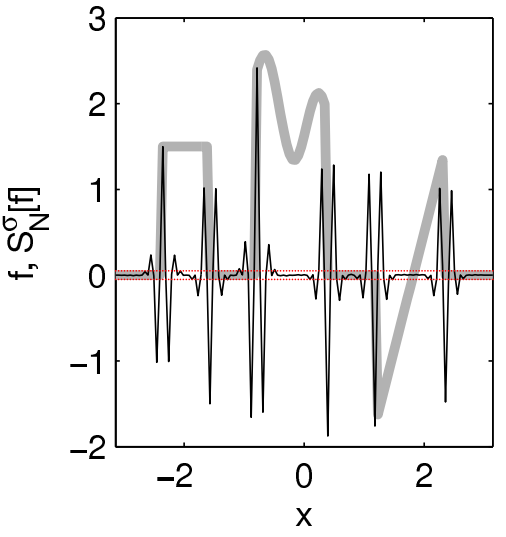

(c) Exponential $\left(\sigma_{\exp }\right)$

Figure 2: Edge detection using different CFs. The test function, (7), is plotted as a thick gray line and the jump function approximation as a thin black line. The low order method in panel (a) has relatively large non-zero entries in parts of the function with steep gradients but no oscillations close to the jumps, while the higher order methods in panels (b) and (c) have smaller entries in regions with steep gradients, but have oscillations close to the jumps. Here $N=64$. 


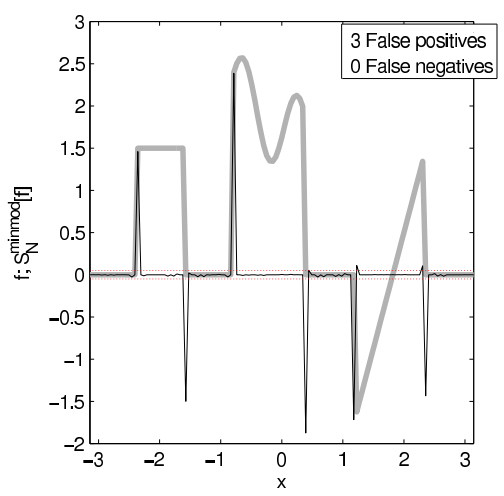

(a) Case 1: Minmod edge detection

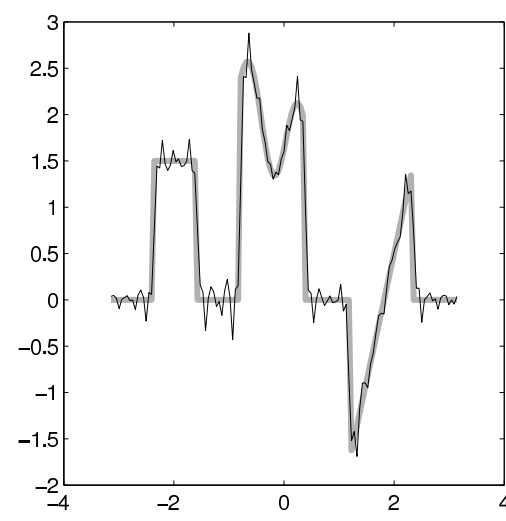

(b) Case 2: 10\% Fourier coefficients missing

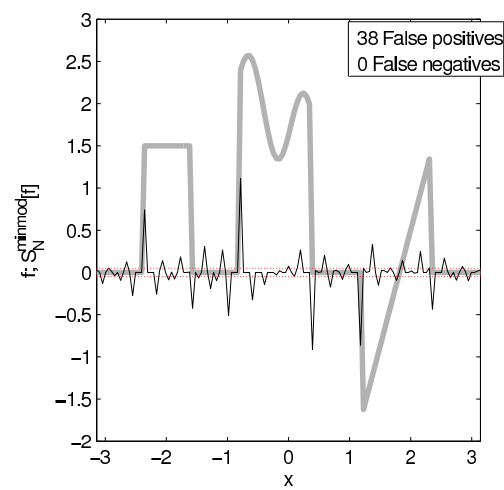

(e) Case 2: Minmod

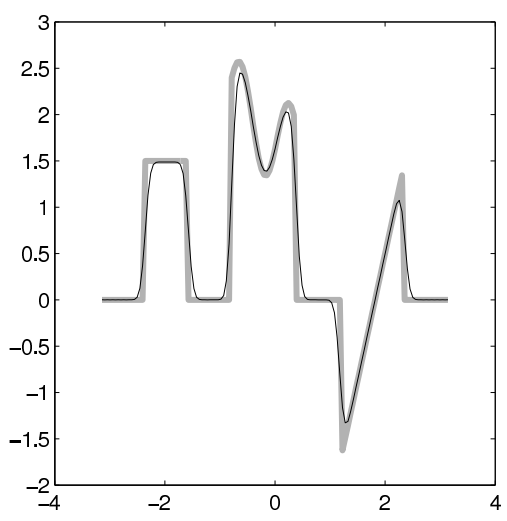

(c) Case 3: Gaussian blur

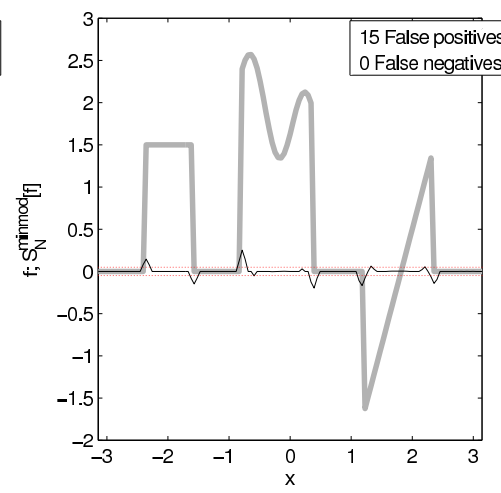

(f) Case 3: Minmod

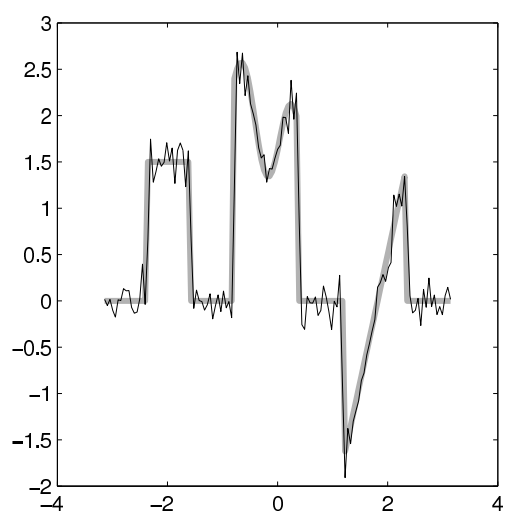

(d) Case 4: Additive noise

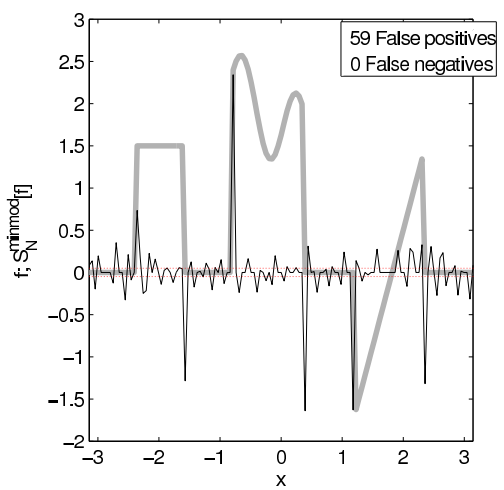

(g) Case 4: Minmod

Figure 3: Panel (a): Minmod post-processing, (9), is applied to the jump function reconstructions in Figure 2 in an attempt to remove unwanted oscillations. Panels (b)-(d): One example for each of the cases 2-4 applied to the test function, (7). The plots shows the partial Fourier sum for the given Fourier coefficients. Panels (e)-(g) show that the minmod method produces many false edges. Here $N=64$. 
to (4) using different CFs. Hence the final result is

$$
S_{N}^{M M}[f](x)=\operatorname{minmod}\left\{S_{N}^{\sigma_{1}}[f](x), S_{N}^{\sigma_{2}}[f](x), \ldots, S_{N}^{\sigma_{n}}[f](x)\right\} .
$$

Figure 3(a) shows the results using (9) by combining the three results in Figure 2. In these experiments, and throughout, the coefficients of blur $\hat{h}_{k}$ and noise $\hat{n}_{k}$ in $(3)$ are given by $\hat{h}_{k}=$ $\exp \left(\left(-k^{2} \tau^{2}\right) / 2\right)$ with $\hat{n}_{k}$ drawn from $C N\left(0, \nu^{2}\right)^{2}$, respectively. Moreover, throughout the paper when results for a single case of blur and/or noise are presented, the parameters chosen are $\tau=.05$ and $\nu=.015$. To count the number of false positive and negative jump detections, we apply an absolute uniform threshold of 0.05 to the absolute value of the jump function approximation. While this choice is somewhat arbitrary, and the performance of the minmod does depend on this threshold, this particular choice appropriately illustrates the impact of the minmod method. In particular, the minmod method works well if a full (finite) set of uncontaminated Fourier coefficients is available, as illustrated in Panel (a). As is evident in this figure, it is the nonlinear combination of the different CFs approximations of various orders that enables its success. Using any individual jump function approximation, whether based on a high or low order CF, would result in more false positive and negative jump detections. Panels (e)-(g) show the results for cases 2-4, where the minmod method fails to provide accurate edge detection results, demonstrating the need to modify the method when Fourier coefficients are missing, or if blurring or noise is present ${ }^{3}$.

\subsection{Matching waveform of the CF method}

In order to improve the $\mathrm{CF}$ method, we first analyze the structure of the oscillations around the jump discontinuities. Following the derivation in [9], we assume a periodic piecewise smooth function $f(x)$ with a single jump discontinuity at $x=\xi$. The jump function approximation for a given $\mathrm{CF} \sigma(\eta)$ is

$$
S_{N}^{\sigma}[f](x)=\frac{[f](\xi)}{\pi} \sum_{k=1}^{N} \sigma\left(\frac{k}{N}\right) \frac{\cos k(x-\xi)}{k}+\mathcal{O}\left(\frac{\log N}{N}\right) .
$$

Hence we see that for large $N$, the jump function approximation depends only on the size and the location of the jump, but not on the function itself. This formulation was exploited in [8], where the authors introduced a family of CFs based on the definition of the matching waveform

$$
W_{N}^{\sigma}(x)=\sum_{k=1}^{N} \sigma\left(\frac{k}{N}\right) \frac{\cos k x}{k} .
$$

Specifically, convolution is used to determine the strongest correlation between the waveform produced by the CF method and the one that results from applying the CF method to an indicator function, yielding (see [8] for details)

$$
S_{N}^{\sigma_{\mathrm{mw}}}[f](x)=\frac{1}{\gamma_{\mathrm{mw}}}\left(S_{N}^{\sigma}[f] * W_{N}^{\sigma}\right)(x) .
$$

\footnotetext{
${ }^{2}$ Here $C N\left(0, \nu^{2}\right)$ denotes the complex normal distribution with mean 0 and variance $\nu^{2}$.

${ }^{3}$ In [29], it is shown that it is possible to construct concentration factors when Fourier data is missing.
} 
Here $\gamma_{\mathrm{mw}}=\frac{1}{\pi} \sum_{k=1}^{N}\left(\frac{\sigma\left(\frac{k}{N}\right)}{k}\right)^{2}$ is used to normalize the approximation. It is straightforward to show that (11) can be rewritten as (4), with $\sigma_{\mathrm{mw}}\left(\frac{|k|}{N}\right)$ an admissible $\mathrm{CF}$ defined as

$$
\sigma_{\mathrm{mw}}\left(\frac{|k|}{N}\right):=\frac{1}{\gamma_{\mathrm{mw}}} \sigma\left(\frac{|k|}{N}\right) \int_{-\pi}^{\pi} W_{N}^{\sigma}(\rho) \exp (-i k \rho) d \rho
$$

While (11) performs better in the presence of noise, the corresponding kernel $C_{N}^{\sigma}$ in (4) is still oscillatory, and hence there are still oscillations in the jump function approximation. Its performance also deteriorates when more jump discontinuities are present, as they start to interfere with the approximation of nearby jumps. However, this approach is useful in constructing the minimization method that we develop in Section 3.

\section{Edge detection in blurred signals by means of $l_{1}$ minimization}

\subsection{The variational approach}

We propose to remove the oscillations and at the same time remove the blur by means of an $l_{1}$ minimization. Based on our previous discussion of the matching waveform and in particular, (11), we seek a sparse spike train $y$ such that

$$
W_{N}^{\sigma} * y \approx S_{N}^{\sigma}[f]
$$

More flexibility is built into (13), however. Clearly, we cannot expect to replace $y$ directly with $[f](x)$, because $[f](x)$ is bounded and has support on a set of measure zero, meaning that the left hand side of (13) would be exactly zero. However, we can expect $y$ to approximate a spike train, which is zero away from the jumps and non-zero at the jump positions (e.g. in (11), $y=\frac{1}{\gamma_{\mathrm{mw}}} S_{N}^{\sigma}[f]$.) Without loss of generality we assume that $f$ has a single jump at $x=0$. If $y$ in (13) is replaced by the approximation of delta distribution

$$
\delta_{N}(x)=[f](0) \frac{2 N+1}{2 \pi}\left\{\begin{array}{lll}
1 & \text { for } & |x| \leq \frac{\pi}{2 N+1} \\
0 & \text { for } & \text { otherwise }
\end{array}\right.
$$

the left hand side of (13) becomes

$$
\begin{aligned}
W_{N}^{\sigma} * \delta_{N}(x) & =\int_{-\pi}^{\pi} W_{N}^{\sigma}(\nu) \delta_{N}(\pi-\nu) d \nu \\
& =W_{N}^{\sigma}(\xi) \int_{-\Delta x}^{\Delta x} \delta_{N}(\pi-\nu) d \nu \\
& =[f](0) W_{N}^{\sigma}(\xi),
\end{aligned}
$$

for some $\xi \in[-\Delta x, \Delta x]$ and $\Delta x=\frac{2 \pi}{2 N+1}$. Thus by setting $y=\delta_{N}(x)$ the approximation holds with equality in the limit for a single jump at the origin. Therefore we conclude that $W_{N}^{\sigma} * y$ is a good approximation of $S_{N}^{\sigma}[f]$ for $N \rightarrow \infty$, if $y$ is a spike train which is zero away from the jump locations and non-zero at the jump locations. However for $N<\infty$, equality can not be required because we seek a sparse representation of a function that identifies the jump locations which does not necessarily follow an equality constraint (e.g. (11) is not sparse). 
Next we derive the discrete $l_{1}$ minimization problem that is solved in the numerical implementation in Section 3.2. From (4) we see that the concentration kernel $C_{N}^{\sigma}$ has the Fourier coefficients

$$
\left(\widehat{C_{N}^{\sigma}}\right)_{k}=i \sigma\left(\frac{|k|}{N}\right) \operatorname{sgn}(k)
$$

The Fourier transform of the matching waveform $W_{N}^{\sigma},(10)$, is given by

$$
\begin{aligned}
\left(\widehat{W_{N}^{\sigma}}\right)_{k} & =\int_{-\pi}^{\pi} W_{N}(x) e^{-i k x} d x \\
& =\int_{-\pi}^{\pi} \sum_{l=1}^{N} \sigma\left(\frac{l}{N}\right) \frac{\cos (l x)}{l} e^{-i k x} d x \\
& =\sum_{l=1}^{N} \frac{\sigma\left(\frac{l}{N}\right)}{l} \int_{-\pi}^{\pi} \cos (l x) e^{-i k x} d x \\
& =\sum_{l=1}^{N} \frac{\sigma\left(\frac{l}{N}\right)}{l}\left\{\begin{array}{rll}
\pi & \text { for } \quad|k|=l \neq 0 \\
0 & \text { otherwise }
\end{array}\right. \\
& =\left\{\begin{array}{rll}
\frac{\pi}{|k|} \sigma\left(\frac{|k|}{N}\right) & \text { for } \quad|k| \leq N, \quad k \neq 0 \\
0 & \text { for } \quad \text { otherwise }
\end{array},\right.
\end{aligned}
$$

and therefore applying $S_{N}^{\sigma}$ to (3) yields

$$
\begin{aligned}
\left(\widehat{S_{N}^{\sigma}[g]}\right)_{k} & =i \sigma\left(\frac{|k|}{N}\right) \operatorname{sgn}(k) \hat{g}_{k} \\
& =\left(\widehat{C_{N}^{\sigma}}\right)_{k} \hat{g}_{k} \\
& \approx\left(\widehat{C_{N}^{\sigma}}\right)_{k} \hat{f}_{k} \hat{h}_{k} \\
& =\hat{h}_{k}\left(\widehat{C_{N}^{\sigma}}\right)_{k} \hat{f}_{k} \\
& \approx \hat{h}_{k}\left(\widehat{W_{N}^{\sigma}}\right)_{k} \hat{y}_{k} \\
& =\hat{h}_{k} \frac{\pi}{|k|} \sigma\left(\frac{|k|}{N}\right) \hat{y}_{k},
\end{aligned}
$$

where $\hat{y}_{k}$ denotes the $k$-th Fourier coefficient of the spike train $y$. Between lines one and two we use (14), between lines two and three we apply (3) without the noise term, and between lines four and five we employ (13). Note that if an equality constraint would be imposed in line 5 then the equation can be multiplied by $\sigma\left(\frac{|k|}{N}\right)$ and $y$ would be independent of the CF. Our goal is to relax the equality in (13) enough so that a sparse function $y$ fits the given data in the presence of noise.

It is known that minimization with respect to the $l_{1}$ norm yields a sparse solution with very high probability, if the restricted isometry property (RIP) is satisfied $[3,4,2]$. It is very difficult to verify the RIP of $l_{1}$ minimization problems except for very few special cases. Even though it is beyond the scope of this paper to prove that the RIP holds, our numerical experiments in section 3.2 indicate that the solution of the minimization problem is stable. For numerical computations we first replace $\left\{\hat{y}_{k}\right\}_{k=-N}^{N}$ in (15) by the discrete Fourier coefficients $\left\{\hat{\mathbf{y}}_{k}\right\}_{k=-N}^{N}$. Assume an equally spaced reconstruction grid

$$
x_{j}=\frac{j \pi}{N}-\pi \quad \text { with } \quad j=0, \ldots, 2 N-1,
$$

and let

$$
\hat{\mathbf{y}}_{k}=\frac{1}{2 N} \sum_{j=0}^{2 N-1} \mathbf{y}_{j} e^{-i k x_{j}}
$$


be the discrete Fourier coefficients ${ }^{4}$ of $\mathbf{y}$ for $k=-N, \ldots, N-1$ and $\hat{\mathbf{f}}=F \mathbf{f}$, where $F \in \mathbb{R}^{(2 N) \times(2 N)}$, such that

$$
F_{k j}=\frac{1}{2 N}(-1)^{k} \exp \left(\frac{-i \pi j k}{N}\right) .
$$

We find the discrete approximation of $y$ by solving

$$
\mathbf{y}=\arg \min _{\mathbf{u}}\|\mathbf{u}\|_{1} \quad \text { subject to } \quad \sum_{k=-N}^{N-1} \sigma\left(\frac{|k|}{N}\right)^{2}\left|\left(\frac{\pi}{|k|} \hat{h}_{k} \hat{\mathbf{u}}_{k}\right)-i \operatorname{sgn}(k) \hat{g}_{k}\right|^{2}<\delta,
$$

where $\delta>0$ is a regularization parameter. Let

$$
\Sigma=\operatorname{diag}\left(\sigma\left(\frac{|-N|}{N}\right), \cdots, 0, \cdots, \sigma\left(\frac{|N-1|}{N}\right)\right)
$$

and suppose $H=\operatorname{diag}\left(\frac{\pi}{|-N|} \hat{h}_{-N}, \cdots, 0, \cdots, \frac{\pi}{|N-1|} \hat{h}_{N-1}\right)$, then (17) has the form

$$
\mathbf{y}=\arg \min _{\mathbf{u}}\|\mathbf{u}\|_{1} \quad \text { subject to } \quad\|\Sigma(H F \mathbf{u}-\mathbf{b})\|_{2}^{2} \leq \delta,
$$

where $\mathbf{b}=\left(-i \hat{g}_{-N}, \cdots, 0, \cdots, i \hat{g}_{N-1}\right)$. This problem can be formulated as a second order cone problem (SOCP) and solved by barrier function or dual interior point methods [14, 13, 24, 27]. Alternatively a Lagrange multiplier $\lambda$ can be introduced yielding

$$
\mathbf{y}=\arg \min _{\mathbf{u}}\left\{\lambda\|\mathbf{u}\|_{1}+\frac{1}{2}\|\Sigma(H F \mathbf{u}-\mathbf{b})\|_{2}^{2}\right\},
$$

which can be efficiently solved by operator splitting methods (see e.g. [30]). Here we are mainly interested in introducing this new method and not about an optimal implementation. We also note that in this formulation $\lambda$ takes over the role of the regularization parameter $\delta$ in (17).

\subsection{Numerical experiments}

We consider cases 2-4 again, where the classical minmod method fails to accurately determine the edge locations of the test function, (7). To solve the edge detection problem with our proposed method, we solve (19) using the CVX software package [13] under MATLAB ${ }^{\circledR}$.

First we consider case 2 with $N=64$ and Fourier coefficients missing from the middle of the spectrum. To determine the jump locations we again apply a constant, uniform threshold of 0.05 to the output $\mathbf{y}$ of (19). Locations where the result is above the threshold are counted as detected edge locations. The detected edge locations are compared to the true edge locations as we count false negatives (missed jumps) and false positives.

Figure 4 shows the edge detection results for various regularization parameters $\lambda$ (x-axis) as the percentage of available Fourier data decreases (y-axis). The number of false positive counts is illustrated as the gray level of the horizontal lines while the number of false negative counts are illustrated as gray levels of the vertical lines. There are four regions in the plot labeled by (a),(b),(c) and (d). Region (a), with only vertical lines, corresponds to solutions of the edge detection problem where false edges are detected. The false detections originate from two sources.

\footnotetext{
${ }^{4}$ Because we use an even number of grid points, (4) is also taken over an even number of discrete Fourier coefficients resulting in $N-1$ for the upper bound of the sum in the discrete case.
} 
First, the deconvolution problem is very ill posed and therefore small errors are amplified, and second, there are errors in $S_{N}^{\sigma}[f],(4)$, as for example in regions with steep gradients. These errors can be controlled by the size of the regularization parameter, which is illustrated in the figure by the decreasing gray values of the vertical lines from left to right. The plot labeled (a) on the right hand side shows one example of the approximation (20) in this region. Clearly false positive edge detection occurs in the steep parts of the test signal. These false positives are due to the error introduced by the assumption that the CF jump function approximation, $S_{N}^{\sigma}[f]$, is equivalent to the sparse spike train, $\mathbf{y}$, convolved with the watching waveform, $W_{N}^{\sigma}$. That is, there is an equality sign in (13). This assumption implies in particular that the steep parts of the test function should result in zero-values in $S_{N}^{\sigma}[f]$ away from the jumps, which is clearly not the case for $N<\infty$, and can be easily be seen from (5). Therefore false positive jumps are detected in the steep regions of the signal. The false positive detections decrease with larger $N$, which is demonstrated in later figures. Region (b) in the plot on the left hand side contains the results of (20) when the regularization parameter is too large. The solutions are over-regularized, so that true jumps are missed. The plot labeled (b) on the right side shows that $\mathbf{y}$ is virtually zero, i.e., all six true jumps are missed. Both plots (a) and (b) on the right hand side are produced with $30 \%$ of the available Fourier coefficients removed. If the regularization parameter is chosen between about $10^{-7}$ and about $10^{-4}$ all jumps are identified correctly with no false positive or negative detections, which is also true for all other points in the region (d). Region (c) illustrates that if there are not enough Fourier coefficients available, then both false positive and false negative detections are present.

Figure 5 summarizes the edge detection results using concentration factors $\sigma_{1}$ and $\sigma_{2}$. Together with Figure 4, the results illustrate the effect that different CFs have on the proposed edge detection method when bands of Fourier data are missing (case 2). In the standard CF method, higher order concentration factors lead to higher order, i.e. faster convergence away from the jump discontinuities. In our proposed inverse formulation, the effects of the higher order CF are less dramatic. In numerical experiments, not shown here, we also observed that higher order concentration kernels can lead to more false jump detections unlike the approach in [9, 10] where higher order kernels generally result in more accurate jump function approximations. This is in part due to the oscillatory responses near the jump discontinuities. ${ }^{5}$ The effect is also likely a consequence of the ill posedness of the deconvolution problem, in particular for the higher order kernels. The RIP and the size of associated constant $\delta_{s}$ which determines the accuracy of the $l_{1}$ solution might play a role as well, see [2] for details. Further investigation is necessary to fully understand this phenomenon. However, the figures show a clear advantage, in the sense that a much larger range of $\lambda$-values lead to zero false edge detections (white regions), of $\sigma_{2}$, in Figure $5(\mathrm{~b})$, and $\sigma_{\exp }$, Figure 4 , over $\sigma_{1}$, in Figure 5(a).

Figure 6 summarizes edge detection results using $\sigma_{\exp }$ with $N=32$ and $N=128$. Figures 4 and 6 illustrate that more Fourier data improves the method's ability to detect the true edges without increasing the number of false positives.

To summarize case 2, the proposed method is able to recover the jump positions when the classical CF method fails, see Figure 3(e). In particular, notice that the CF method fails even when $90 \%$ of the Fourier data is available. We note that concentration factors were specifically designed for the missing bandwidth case in $[28,29]$.

\footnotetext{
${ }^{5}$ In [5] a concentration kernel was designed to enforce a more uniform approximation error without oscillations near the jumps. While not as effective for functions with a lot of variation between jumps, the kernel is well suited for noisy data. Future investigations will incorporate this kernel into the variational approach developed here.
} 

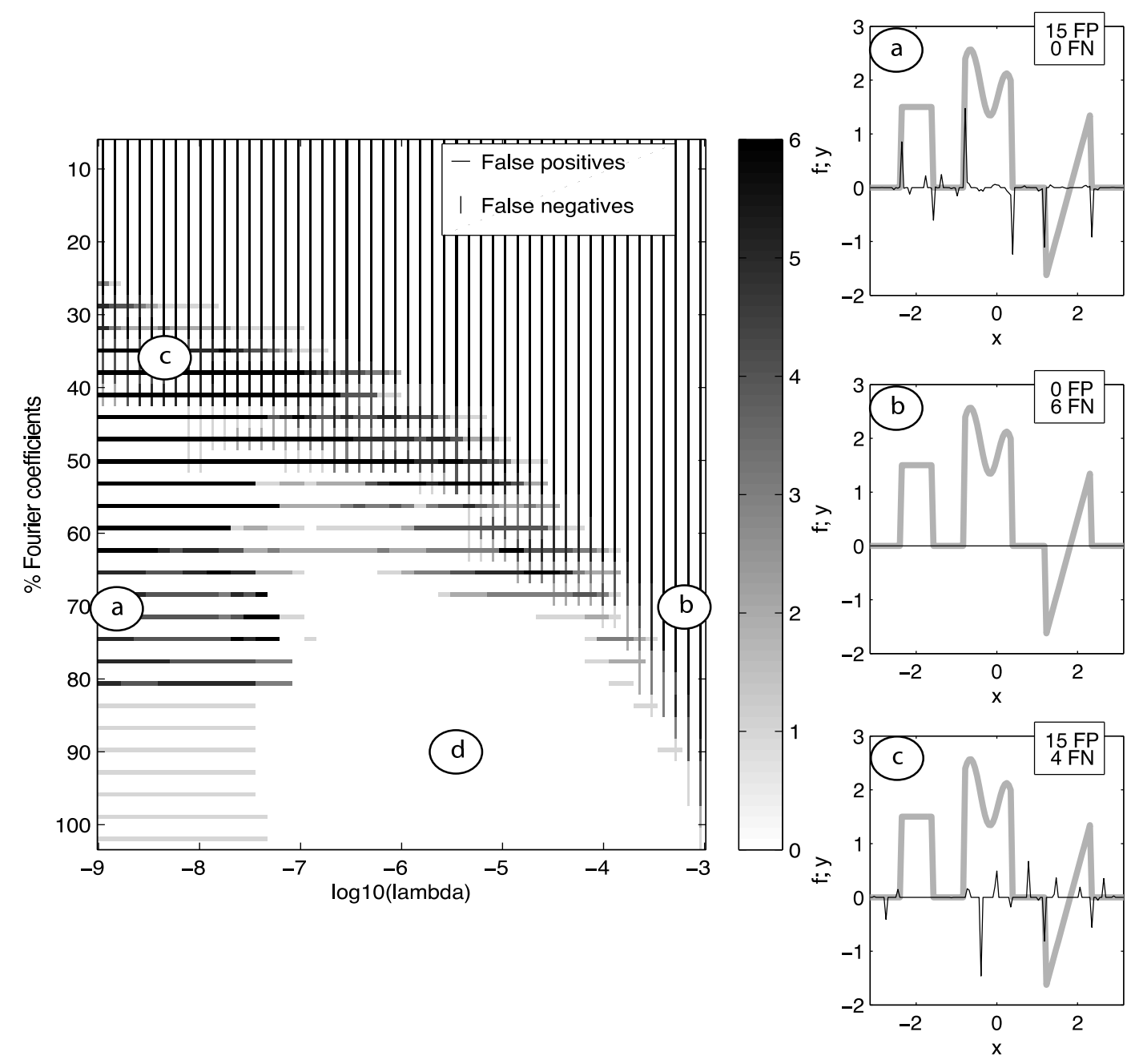

Figure 4: Erroneous edge detection results for case 2 using $\sigma_{\exp }$. In region (a) false positive edge detections are present, in region (b) false negative edge detections and in region (c) both false positive and negative edge detections. In region (d) the edge detection problem is solved without false counts. Here $N=64$. 


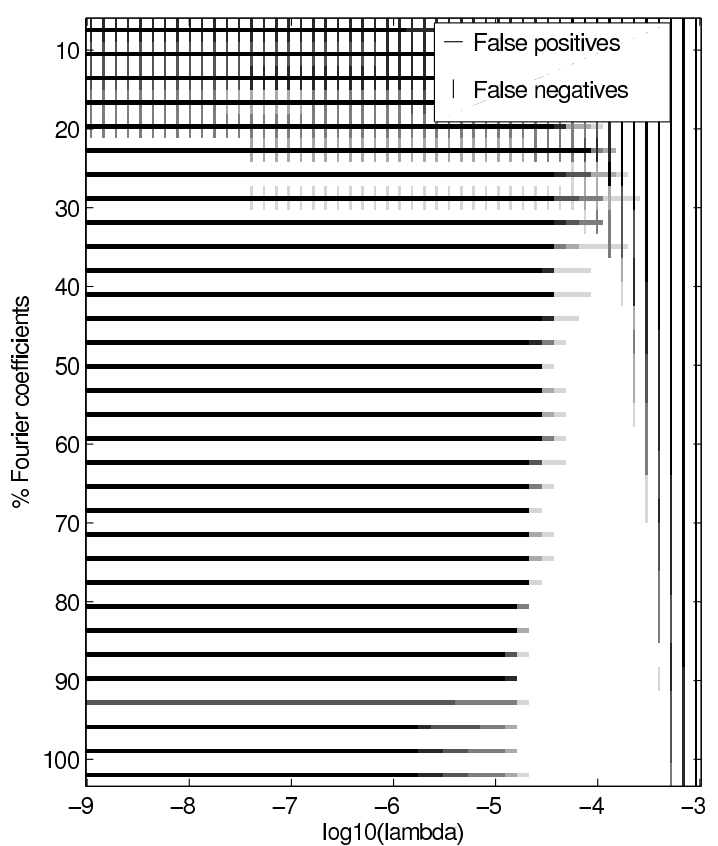

(a) Case 2, $\mathrm{N}=64, \sigma_{1}$
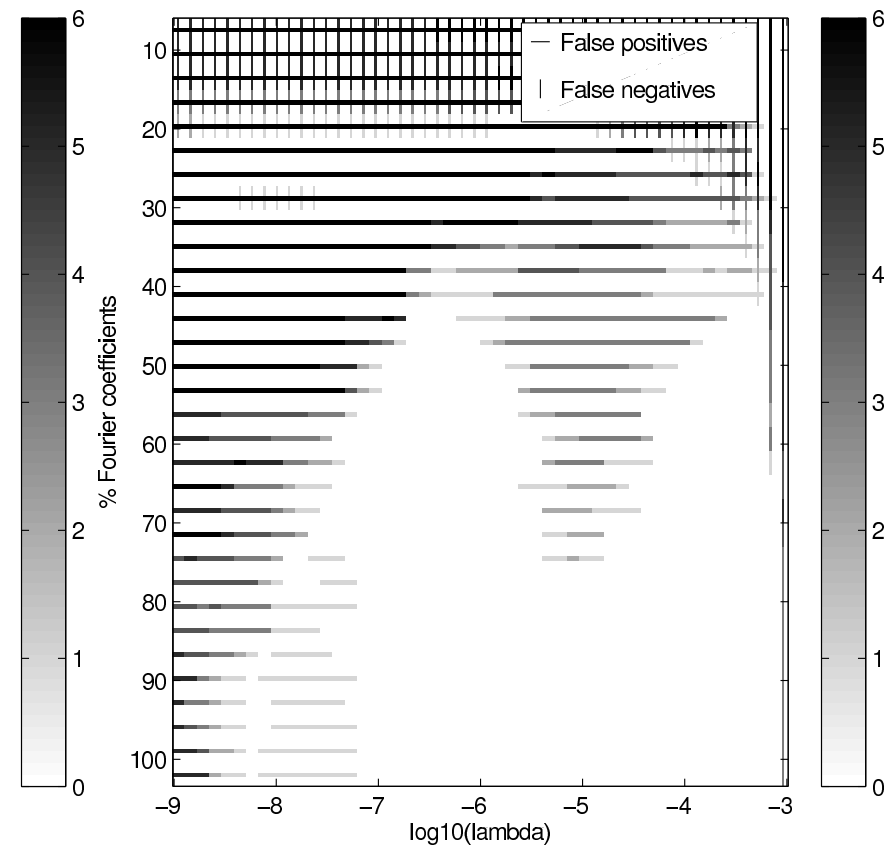

(b) Case 2, N=64, $\sigma_{2}$

Figure 5: Case 2: Edge detection on undersampled data, polynomial concentration factors. The $y$-axis is the percentage of Fourier coefficients available. Here $N=64$.

Figure 7 shows edge detection results for case 3, where the Fourier coefficients are contaminated by blur with $\hat{h}_{k}=\exp \left(-\left(k^{2} \tau^{2}\right) / 2\right)$, and the range for $\tau$ is indicated on the $y$-axis. Notice that application of the blur represents filtering of the coefficients. Likewise, the case of missing Fourier coefficients, case 2, corresponds to a filter, but with a band pass indicator function in the Fourier domain. The results demonstrate that the proposed method can handle blurring where the classical CF method fails under the same level of blur, compare to Figure 3(f). As in case 2, we observe that the higher order CFs perform better than the lower order $\mathrm{CF}$, which is indicated by the larger white regions in Panel (b) and (c) as compared to Panel (a).

Finally, we consider case 4 where the Fourier coefficients are contaminated by noise. These results are illustrated in Figure 8 and show that our proposed method can handle noise, where the classical CF method fails. For either $\sigma_{1}$ and $\sigma_{\text {exp }}$, a regularization parameter $\lambda$ can be found which correctly detects all edges at a noise level of $\nu=.015$. Recall that the CF method fails in this case. In fact, Figure $3(\mathrm{~g})$ shows many false positive detections. In this case $\sigma_{\exp }$ performs better than $\sigma_{2}$, which is probably a consequence or the high frequency filtering associated with the exponential $\mathrm{CF}$.

\section{Extension to non-harmonic Fourier data}

In this section we are interested in the detection of jump discontinuities given non-harmonic or nonuniform Fourier measurements. This is a problem of interest in applications such as MR imaging, where it has been empirically observed that images processed from non-Cartesian spectral 


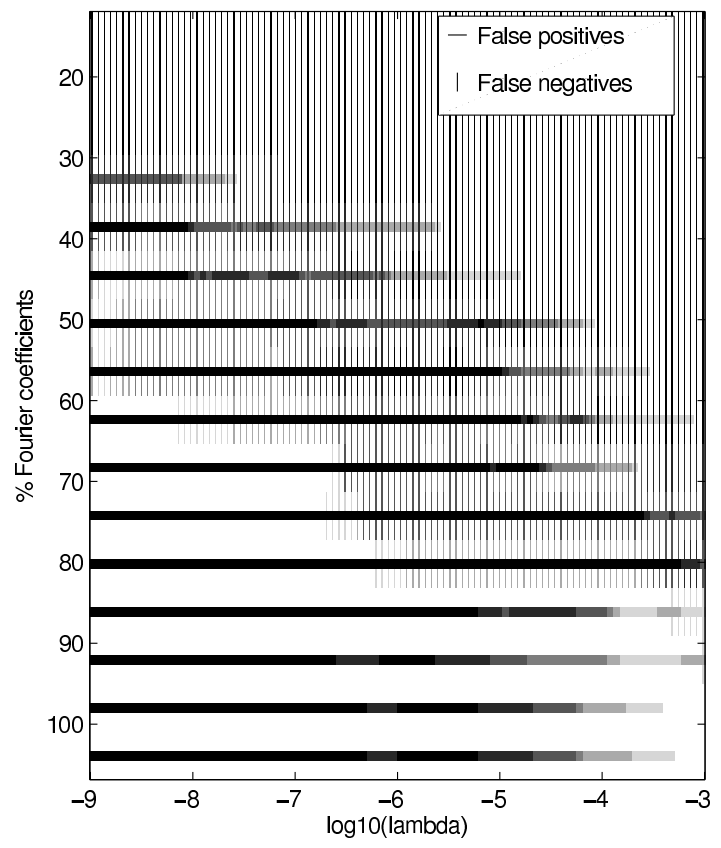

(a) Case $2, N=32$

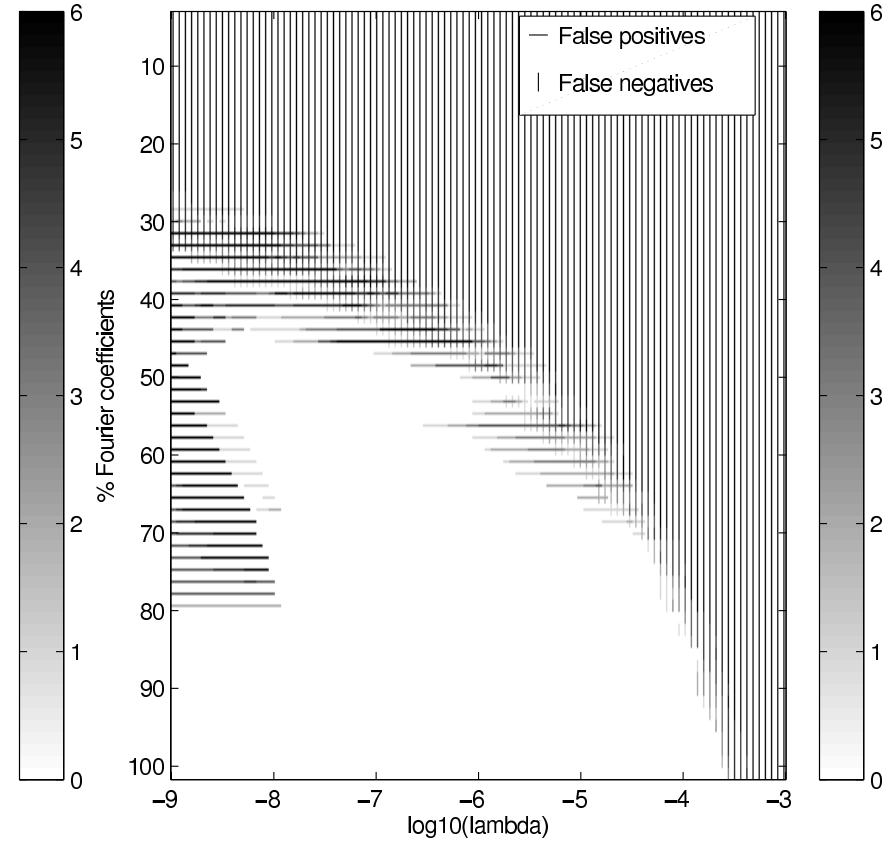

(b) Case 2, $N=128$

Figure 6: Case 2: Edge detection on undersampled data using $\sigma_{\text {exp }}$. The $y$-axis is the percentage of Fourier coefficients available.

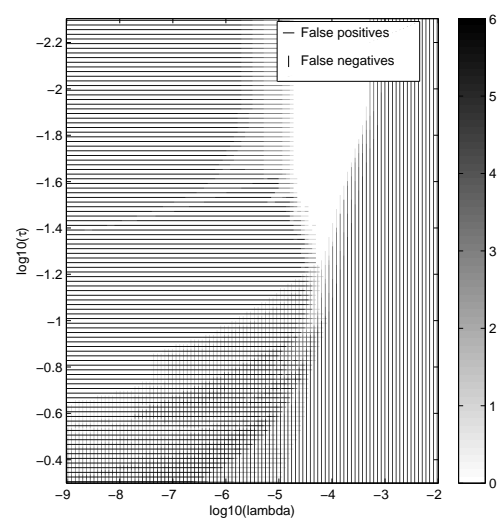

(a) $\sigma_{1}$

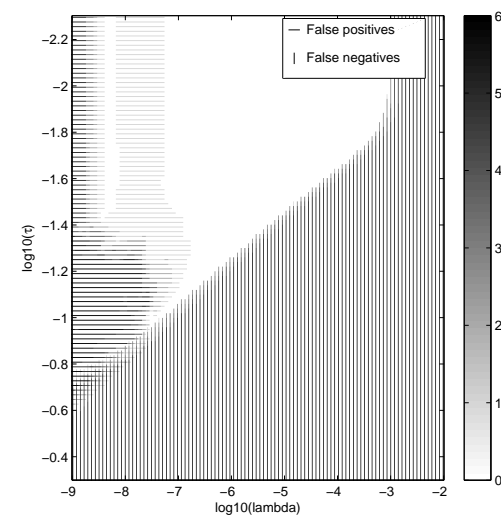

(b) $\sigma_{2}$

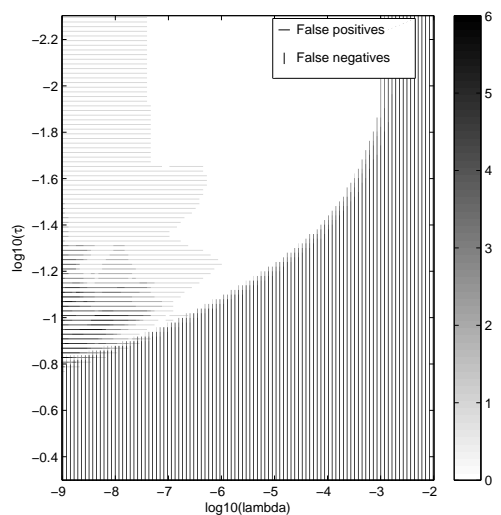

(c) $\sigma_{\exp }$

Figure 7: Case 3: Edge detection for blurred signals. The $y$-axis indicates the extent of the blur as measured by $\log 10(\tau)$. Here $N=64$. 


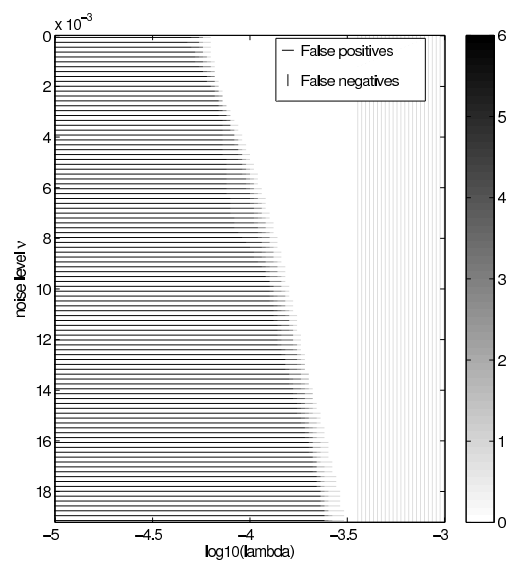

(a) $\sigma_{1}$

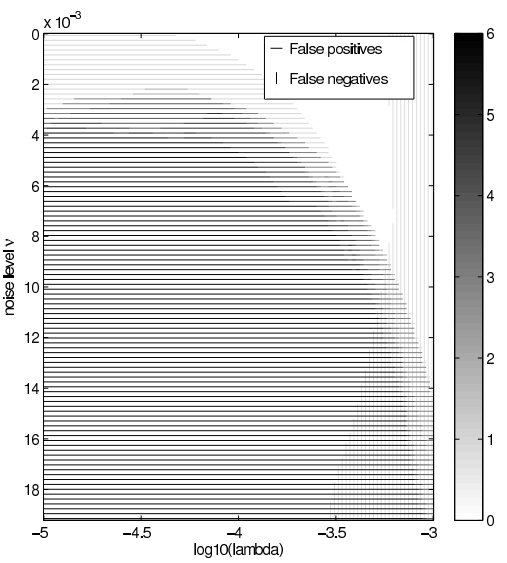

(b) $\sigma_{2}$

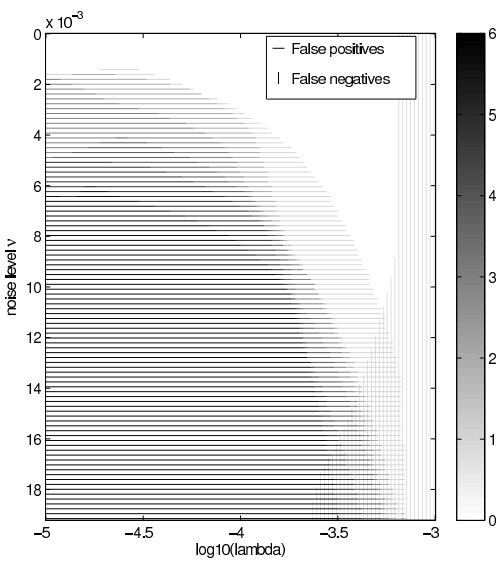

(c) $\sigma_{\exp }$

Figure 8: Case 4: Edge detection for noise contaminated Fourier coefficients. The $y$-axis indicates standard deviation $\nu$ for noise $\hat{n}_{k} \in C N\left(0, \nu^{2}\right)$. Here $N=64$.

data have improved robustness to aliasing and motion artifacts. Non-harmonic Fourier reconstruction, however, is a challenging problem, [31], with the reconstruction quality depending on the set of measurement modes. The acquisition of data at nonuniform sampling densities, for example, presents complications to the reconstruction process.

Definition 1 (Non-harmonic Fourier Data) Let $f \in L^{2}(\mathbb{R})$ be a piecewise-analytic function supported in $(-\pi, \pi)$ and zero elsewhere on the real line. We define non-harmonic Fourier data, $\hat{f}\left(\omega_{k}\right)$, to be the inner-products of $f$ with the non-harmonic exponentials $\left\{e^{i \omega_{k} x}\right\}, \omega_{k} \notin \mathbb{Z}$, i.e.,

$$
\hat{f}\left(\omega_{k}\right):=\frac{1}{2 \pi} \int_{-\pi}^{\pi} f(x) e^{-i \omega_{k} x} d x, \omega_{k} \notin \mathbb{Z} .
$$

We now present a formulation of the concentration method applicable to non-harmonic Fourier data. Consider a compactly supported function $f$. For ease of analysis, we assume that there are no jumps near the ends of the interval. As in (4) we arrive at jump function approximations by convolving $f$ with suitable convolutional kernels ${ }^{6}$, i.e.,

$$
\tilde{S}_{N}^{\sigma}[f](x)=\left(f * \tilde{C}_{N}^{\sigma}\right)(x)
$$

with

$$
\tilde{C}_{N}^{\sigma}(x)= \begin{cases}C_{N}^{\sigma}(x)=i \sum_{k=-N}^{N} \operatorname{sgn}(k) \sigma\left(\frac{|k|}{N}\right) e^{i k x} & |x| \leq \pi \\ 0 & \text { otherwise. }\end{cases}
$$

Since $\tilde{S}_{N}^{\sigma}[f]$ is a compactly supported function, it has a Fourier transform representation with

$$
\widehat{\tilde{S}_{N}^{\sigma}[f]}(\omega)=\hat{f}(\omega) \widehat{\tilde{C}_{N}^{\sigma}}(\omega) .
$$

\footnotetext{
${ }^{6}$ We use a superscript $\sim$ to distinguish quantities computed from non-harmonic Fourier data from their standard Fourier equivalents.
} 
The Fourier coefficients of the concentration kernel, $\tilde{C}_{N}^{\sigma}(x)$ are given by

$$
\begin{aligned}
\widehat{\tilde{C}}_{N}^{\sigma}(\omega) & =\left.\mathcal{F}\left\{\tilde{C}_{N}^{\sigma}(x) \Pi\left(\frac{x}{2 \pi}\right)\right\}\right|_{\omega} \\
& =\left(\hat{C}_{N}^{\sigma} * \operatorname{sinc}\right)(\omega) \\
& =\sum_{k=-\infty}^{\infty} \hat{C}_{N}^{\sigma}(k) \operatorname{sinc}(\omega-k) .
\end{aligned}
$$

Here $\Pi(x)$ denotes the rectangular window function of width $2 \pi$ and height 1 .

The Fourier coefficients of the concentration jump function approximation corresponding to a set of non-harmonic measurements, $\hat{f}\left(\omega_{k}\right), k=-N, \ldots, N$, may then be computed using (24). We are then left with the task of recovering $\tilde{S}_{N}^{\sigma}[f]$ from its non-harmonic coefficients. Standard Fourier formulations, such as the concentration sum of (4) are no longer applicable, since the non-harmonic modes violate the quadrature rules for discrete Fourier expansions. However, the following extension of the concentration sum may be employed to compute jump function approximations in this case:

$$
\tilde{S}_{N}^{\sigma}[f](x):=i \sum_{k=-N}^{N} \beta_{k} \hat{f}\left(\omega_{k}\right) \operatorname{sgn}\left(\omega_{k}\right) \sigma\left(\frac{\left|\omega_{k}\right|}{N}\right) e^{i \omega_{k} x} .
$$

This expression arises from interpreting the Fourier partial sum as a quadrature formula for the inverse Fourier integral. Given non-harmonic Fourier data, we use a nonuniform trapezoidal quadrature and appropriate quadrature weights, $\beta_{k}$. One simple choice for $\beta_{k}$ is the inter-sample distance $\omega_{k+1}-\omega_{k}$, although other methods exist for the selection of these weights. We also note that (26) is no longer amenable to FFT computations on account of the nonuniform input data distribution; nevertheless, it can be computed efficiently using nonuniform FFT schemes [23, 7]. The interested reader is referred to literature from the MR imaging community, where this procedure is known as convolutional gridding, $[18,20]$, and the weights are known as density compensation factors, $[19,16]$.

Illustrative jump function approximations using two representative sets of non-harmonic Fourier data are now provided. The associated sampling distributions are plotted in Fourier space in Figure 9. Figure 9(a) illustrates the jittered sampling pattern, where the non-harmonic modes are obtained by uniformly jittering the equispaced Fourier modes,

$$
\omega_{k}=k \pm \zeta_{k}, \quad \zeta_{k} \sim U[0, \theta], k=-N,-(N-1), \ldots, N .
$$

The $\zeta_{k}$ 's are i.i.d. uniform random variables drawn from $U[0, \theta]$, where $\theta$ represents the maximal jitter. Both positive and negative jitters are equiprobable, with the sign of jitter at each node being independent of the sign of jitter at any other node. Figure 9(b) illustrates the log sampling pattern, where samples are acquired at logarithmic intervals, with more samples acquired in lower frequencies. If $\omega_{k}$ are the nodes at which measurements are acquired, $\left|\omega_{k}\right|$ is logarithmically distributed between $10^{-v}$ and $N$, with $v>0$ and $2 N+1$ being the total number of samples. This sampling scheme is useful in understanding performance when measurements are acquired with variable sample density, such as the spiral, [1], and radial sampling schemes in MR imaging.

We use the following test function to compute simulation results:

$$
f(x)= \begin{cases}\sin (3 x) & -\pi \leq x<-\frac{\pi}{3} \\ \tanh (x) & -\frac{\pi}{3} \leq x<\frac{\pi}{2} \\ -\frac{3 x}{\pi}+3 & \frac{\pi}{2} \leq x<\pi\end{cases}
$$




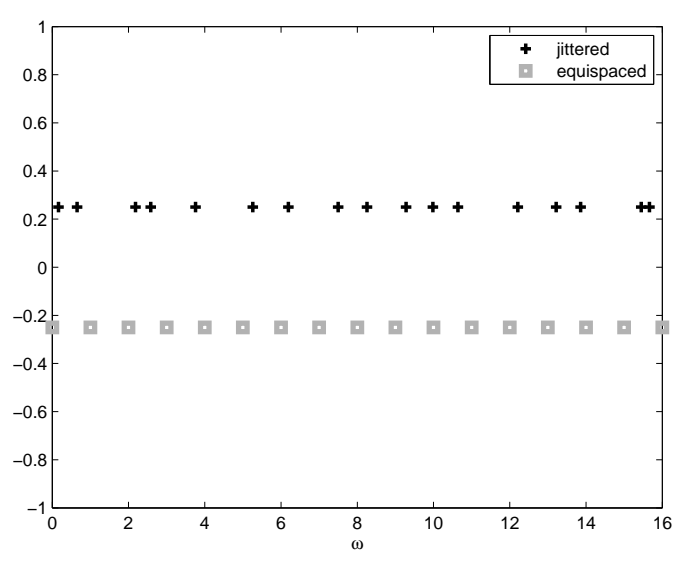

(a) Jittered sampling

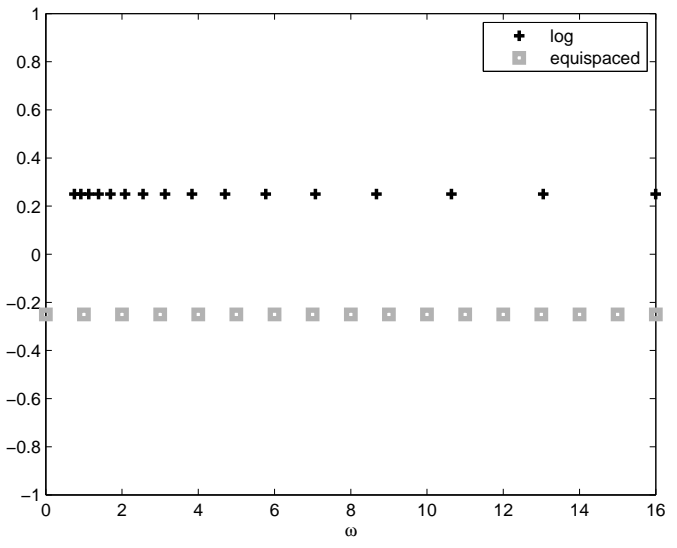

(b) Log sampling

Figure 9: Non-harmonic sampling distributions (right half plane), $N=16$

Its associated jump function is

$$
[f](x)=\left\{\begin{array}{cl}
\tanh \left(-\frac{\pi}{3}\right) \approx-0.7807 & x=-\frac{\pi}{3} \\
1.5-\tanh \left(\frac{\pi}{2}\right) \approx 0.5828 & x=\frac{\pi}{2} \\
0 & \text { otherwise }
\end{array}\right.
$$

We note that this test function has a combination of small jumps and signal variation. In combination with non-harmonic data, blurring and noise, this makes for a particularly challenging jump detection problem. The corresponding jump function approximations for the two sampling schemes are plotted in Figure 10. Figure 10(a) plots the jump function approximation from jittered spectral data using $2 N+1=129$ Fourier modes. Approximations using the first order polynomial factor $\left(\sigma_{1}\right)$ and the exponential concentration factor with $\alpha=1\left(\sigma_{\exp }\right)$ are provided. In both cases, note the presence of artifacts throughout the domain. A comparison with the plots of Figure 2 reveals that the smooth regions, where the response is supposed to be vanishingly small, are filled with spurious responses. Note that these oscillations are not from the concentration method; rather, they are caused by the non-harmonic acquisition. As before, the higher-order factor helps mitigate some of the spurious response in the oscillatory region (away from the jumps). Figure 10(b) plots the corresponding results for log spectral data. Note the relatively poor approximation quality with strong oscillations and poor localization. Simulation results also reveal that the approximation does not converge to the true jump function when using an increased number of measurements.

The underlying cause of these artifacts is the acquisition of non-harmonic spectral data. In particular, families of non-harmonic exponentials $\left\{e^{i \omega_{k} x}\right\}, \omega_{k} \notin \mathbb{Z}$ do not form a basis for functions of practical interest, [31], except under very stringent circumstances ${ }^{7}$. A model for the accurate inversion of such data is difficult to obtain, with the problem compounded by the acquisition of data deviating significantly from the uniform modes. Under these circumstances, variational formulations along the lines of Section 3, which only require an accurate forward model, form an attractive and powerful framework for computing jump function approximations.

\footnotetext{
${ }^{7}$ Kadec's "one-quarter" theorem, [17], allows for reconstruction in the special case that $\sup _{k}\left|\omega_{k}-k\right|<\frac{1}{4}$.
} 


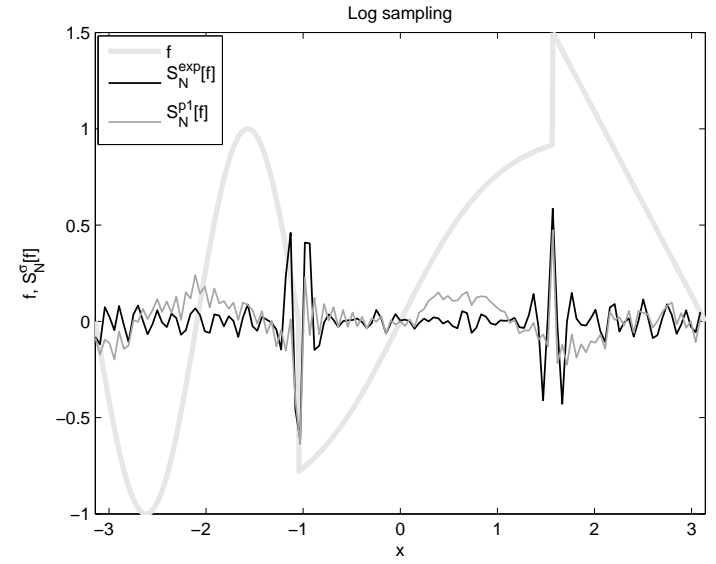

(a) Jittered sampling

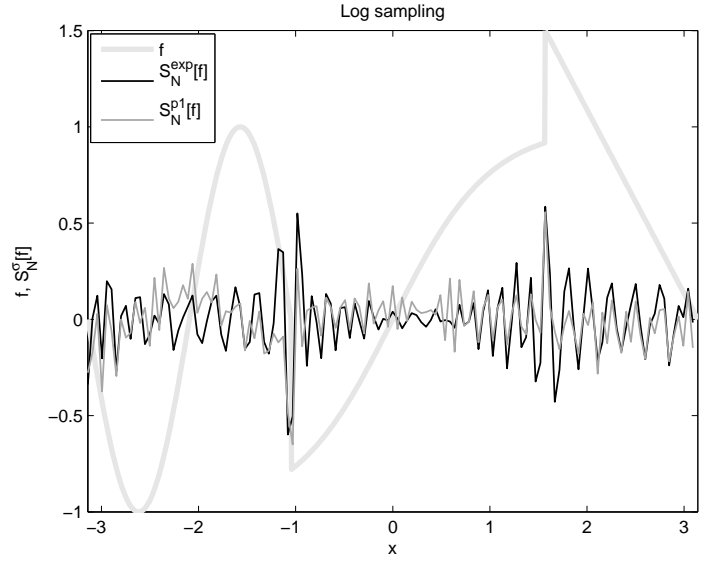

(b) Log sampling

Figure 10: Jump approximations from non-harmonic Fourier data using the non-harmonic concentration sum, $N=64$

\subsection{Variational solutions for non-harmonic Fourier data}

As in Section 3, we solve for a discrete representation, $\mathbf{y}$, of the jump function $[f]$ on an equispaced grid $x_{j}=\frac{\pi j}{N}-\pi, j=0, \ldots, 2 N-1$. Let $\mathbf{g}$ denote the set of non-harmonic measurements of the blurred and possibly noisy Fourier data, i.e., $\mathbf{g}=\left(\hat{g}\left(\omega_{-N}\right), \ldots, \hat{g}\left(\omega_{N-1}\right)\right)^{T}$. Let $\Sigma$ be the diagonal matrix of concentration factors on the non-harmonic modes,

$$
\Sigma=i \operatorname{diag}\left(\operatorname{sgn}\left(\omega_{-N}\right) \sigma\left(\frac{\left|\omega_{-N}\right|}{N}\right), \ldots, 0, \ldots, \operatorname{sgn}\left(\omega_{N-1}\right) \sigma\left(\frac{\left|\omega_{N-1}\right|}{N}\right)\right) .
$$

Let $H$ denote the diagonal matrix of blur coefficients on the non-harmonic modes,

$$
H=\operatorname{diag}\left(\hat{h}\left(\omega_{-N}\right), \ldots, \hat{h}\left(\omega_{N-1}\right)\right) \text {, }
$$

and $F \in \mathbb{C}^{2 N \times 2 N}$ be the discrete non-harmonic Fourier matrix with elements

$$
F_{k j}=\exp \left[i\left(-\pi+\frac{\pi j}{N}\right) \omega_{k}\right], \quad k=-N, \ldots ., N-1, j=0, \ldots, 2 N-1 .
$$

Let $W$ be a Toeplitz matrix whose rows contain shifted replicates of the jump waveform $W_{N}^{\sigma}(x)$, (10). ${ }^{8}$ We may now compute jump approximations by solving

$$
\mathbf{y}=\arg \min _{\mathbf{u}}\|\mathbf{u}\|_{1} \quad \text { subject to } \quad\|H F W \mathbf{u}-\Sigma \mathbf{g}\|_{2}^{2}<\delta .
$$

Alternately, using a Lagrange multiplier, we may write

$$
\mathbf{y}=\arg \min _{\mathbf{u}}\left\{\lambda\|\mathbf{u}\|_{1}+\frac{1}{2}\|H F W \mathbf{u}-\Sigma \mathbf{g}\|_{2}^{2}\right\} .
$$

\footnotetext{
${ }^{8}$ Note that $W_{N}^{\sigma}(x)$ is large only in the immediate vicinity of $x=0$. Hence, $W$ can be well approximated by a banded Toeplitz matrix, reducing the overall computational cost.
} 
As before, this problem can be formulated as a SOCP, whose solution can be computed using one of several methods such as barrier methods or dual interior point methods. Computational efficiency may be improved by computing matrix-vector products involving the non-harmonic Fourier matrix $F$ using nonuniform FFT schemes. The notable distinction from the formulation of Section 3 is the modeling of the jump waveform in physical space for the non-harmonic case. This is necessitated by the discontinuity in the Fourier coefficients of the jump waveform, $\widehat{w_{N}^{\sigma}}(k)$, at $k=0$ (Section $3)$. Interpolating the given discrete data $\hat{f}\left(\omega_{k}\right)$ yields oscillations around $\omega=0$. As a result, there is a model mismatch between the physical space jump waveform, $W_{N}^{\sigma}$, and its corresponding nonharmonic Fourier coefficients. We mitigate the problem by approximating the jump waveform in physical space as a convolution (the Toeplitz matrix $W$ ).

Numerical results using this formulation are provided in Figure 11. Figures 11(a) and (b) plot the jump function approximation of $f(x)$, (4), using $2 N=128$ jittered and log spectral modes respectively. Results for the jittered spectral data are processed using $\sigma_{1}$, while those for the $\log$ spectral data are processed using $\sigma_{\text {exp }}$. We note that the use of low order concentration factors in the log sampling case results in increased false positives, or a higher probability of missed detection of the jump at $x=\frac{\pi}{2}$. Results for both sampling schemes reveal a marked improvement over the quality of the forward methods in Figure 10. The jump function approximations are highly localized and show minimal spurious responses. Regularization parameter values of $1.6 \times 10^{-3}$ and $2 \times 10^{-3}$ respectively were used in the generation of the plots. The variational solutions suffer from incorrect approximation of the jump heights, as this depends on the choice of the regularization parameter $\lambda$. However, once the jump locations are identified by thresholding, the correct jump values may be recovered by a simple evaluation of the concentration sum.

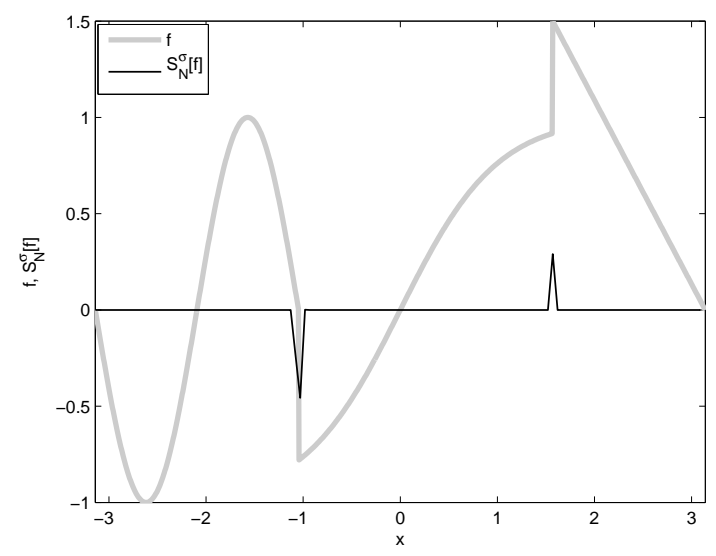

(a) Jittered sampling, $\sigma_{1}$

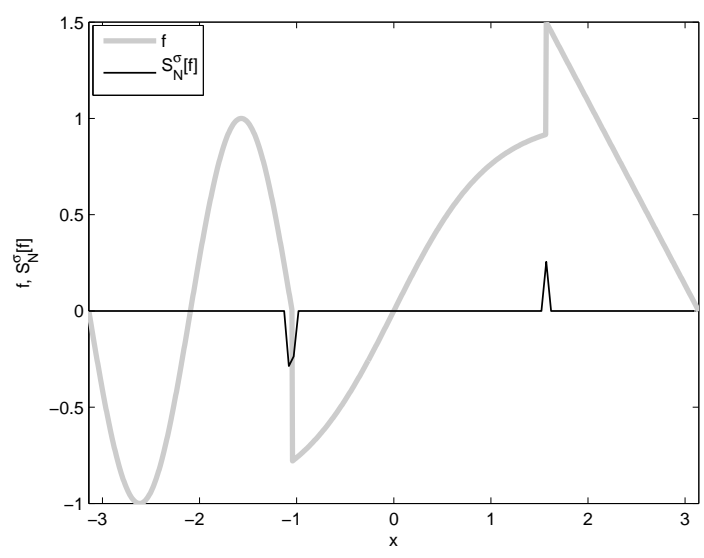

(b) Log sampling, $\sigma_{\exp }$

Figure 11: Jump approximations from non-harmonic Fourier data using the variational formulation, $N=64$

Although exhaustive simulations of the effect of the regularization parameter $\lambda$ on the final solution have not been performed, there are strong indications that parameter selection is more sensitive in the case of non-harmonic Fourier data, as compared to the standard Fourier case. Moreover, the parameter selection problem is more challenging when reconstructing from log spectral samples than jittered samples. 


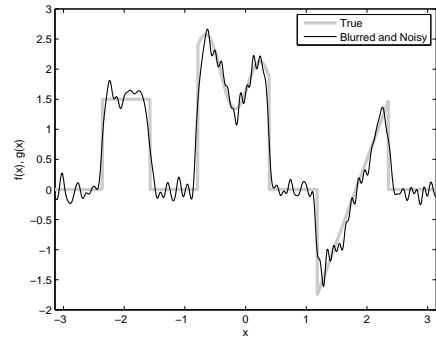

(a) Fourier reconstruction

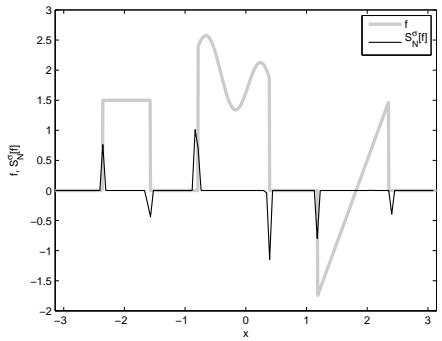

(b) Jittered spectral data, $\sigma_{1}$

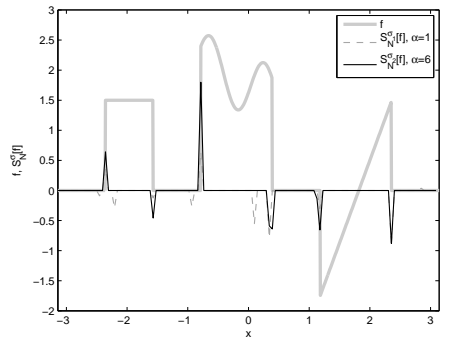

(c) Log spectral data, $\sigma_{\exp }$

Figure 12: Jump detection from blurred and noisy non-harmonic Fourier data, $N=64$

For completeness, representative results of edge detection from blurred and noisy non-harmonic spectral data are provided in Figure 12. A visual illustration of the blur and noise is provided in Figure 12(a), where the Fourier reconstruction of the function is provided. Figures 12(b) and (c) plot the solution of the variational formulation (34) for the jittered and log sampling acquisitions respectively. In either case, the jump function was estimated from $2 N=128$ Fourier coefficients. For jittered data $\sigma_{1}$ was used to compute results, with a regularization parameter value of $\lambda=$ $2.05 \times 10^{-3}$. For the $\log$ sampled data, $\sigma_{\exp }$ with $\alpha$ values of 1 and 6 were used to generate the plots. The corresponding regularization parameter values were $\lambda=1 \times 10^{-3}$ and $1.5 \times 10^{-3}$ respectively. In this case, with few high frequency Fourier measurements available, and corruption by noise and blur, the higher order $\mathrm{CF}$ outperforms the lower order $\mathrm{CF}$, which misses jumps or registers false positives.

\section{Concluding remarks}

We have presented a new approach to detect edges in piecewise smooth functions from partial Fourier data, that might also be corrupted by noise or blurred by a PSF. The method combines the $\mathrm{CF}$ method for edge detection with ideas from compressed sensing. The oscillations introduced by the CF method can be removed by a regularized deconvolution. The method is robust under noise and blurring of the signal, and works even when the Fourier data is given on a nonuniform grid.The exponential CF usually outperform lower order polynomial CFs, in particular when only a limited number of Fourier coefficients are available. Similarly they appear to perform better when the nonuniform Fourier data is sampled more erratically, as in the log-sampling case. Further test cases with fewer jumps and more variation in the test signal confirm this pattern. The method seems to be very promising and more research should be conducted into understanding its limits in the presence of noise and blurring. In particular the method is based on the estimation of the matching waveform, which is derived from a ramp function approximation of general jumps. Therefore we can expect that the accurate edge detection becomes more difficult as the jumps move closer to each other. A more comprehensive computational investigation as well as theoretical results based on the reconstruction probability using the compressive sensing framework, as in [3], should be able to answer this question. A two dimensional extension is possible and subject to a companion paper that is currently in preparation. 


\section{Acknowledgments}

The work of A. Gelb and A. Viswanathan was supported in part by NSF grants DMS 0652833 and DMS 0608844. The work of R. Renaut was supported in part by the NSF grant DMS 0903737. The work of W. Stefan at Rice University was supported in part by NSF Grant DMS-07-48839 and the U. S. Army Research Laboratory and the U. S. Army Research Office grant W911NF-09-1-0383. We would also like to thank Dr. Wotao Yin at Rice University for many fruitful discussions.

\section{References}

[1] C. B. Ahn, J. H. Kim, and Z. H. Cho. High-speed spiral-scan echo planar NMR imaging-I. IEEE Transactions on Medical Imaging, 5(1):2, 1986.

[2] E. Candes, J. Romberg, and T. Tao. Stable signal recovery from incomplete and inaccurate measurements. Communications on Pure and Applied Mathematics, 59(8):1207-1223, 2006.

[3] E. Candes, J. Romberg, and T. Tao. Robust uncertainty principles: Exact signal reconstruction from highly incomplete frequency information. IEEE Transactions on Information Theory, 52(2):489-509, 2006.

[4] E. Candes and T. Tao. Near-optimal signal recovery from random projections: Universal encoding strategies? IEEE Transactions on Information Theory, 52(12):5406-5425, 2006.

[5] D. Cochran, A. Gelb and Y. Wang. Edge detection from truncated Fourier data using spectral mollifiers, preprint.

[6] S. Engelberg and E. Tadmor. Recovery of edges from spectral data with noise - a new perspective. SIAM Journal On Numerical Analysis, 46(5):2620-2635, 2008.

[7] J. A. Fessler and B. P. Sutton. Nonuniform fast Fourier transforms using min-max interpolation. IEEE Transactions on Signal Processing, 51(2):560-574, 2003.

[8] A. Gelb and D. Cates. Detection of edges in spectral data III - refinement of the concentration method. Journal of Scientific Computing, 36(1):1-43, 2008.

[9] A. Gelb and E. Tadmor. Detection of edges in spectral data. Applied and Computational Harmonic Analysis, 7:101-135, 1999.

[10] A. Gelb and E. Tadmor. Detection of edges in spectral data II. nonlinear enhancement. SIAM Journal on Numerical Analysis, 38(4):1389-1408, 2000.

[11] A. Gelb and E. Tadmor. Adaptive edge detectors for piecewise smooth data based on the minmod limiter. Journal of Scientific Computing, 28(2-3):279-306, 2006.

[12] D. Gottlieb and C. W. Shu. On the Gibbs phenomenon and its resolution. SIAM Review, 39(4):644-668, 1997.

[13] M. Grant and S. Boyd. CVX: Matlab software for disciplined convex programming, version $1.21,2010$. 
[14] M. Grant, S. Boyd, and Y. Ye. Disciplined Convex Programming, pages 155-210. Nonconvex Optimization and its Applications. Springer, 2006.

[15] W. Guo and W. Yin. EdgeCS: Edge guided compressive sensing reconstruction. Technical report, Rice CAAM Report TR10-02, 2010.

[16] R. D. Hoge, R. K. Kwan, and G. B. Pike. Density compensation functions for spiral MRI. Magnetic Resonance in Medecine, 38(1):117-28, 1997.

[17] M.I. Kadec. The exact value of the Paley-Wiener constant. Soviet mathematics - Doklady, 5:559-561, 1964.

[18] J. D. O'Sullivan. Fast sinc function gridding algorithm for Fourier inversion in computer tomography. IEEE Transactions on Medical Imaging, 4(4), 1985.

[19] J. G. Pipe and P. Menon. Sampling density compensation in MRI: Rationale and an iterative numerical solution. Magnetic Resonance in Medicine, 41(1):179-186, 1999.

[20] H. Sedarat and D. G. Nishimura. On the optimality of the gridding reconstruction algorithm. IEEE Transactions on Medical Imaging, 19(4):306-317, 2000.

[21] B. Shizgal and J. H. Jung. Towards the resolution of the Gibbs phenomena. Journal of Computational and Applied Mathematics, 161(1):41-65, 2003.

[22] W. Stefan, R. A. Renaut, and A. Gelb. Improved total variation-type regularization using higher-order edge detectors. SIAM Journal on Imaging Sciences, 3(2):232-251, 2010.

[23] G. Steidl. A note on fast Fourier transforms for nonequispaced grids. Advances in Computational Mathematics, 9(3):337-352, 1998.

[24] J. F. Sturm. Using SeDuMi 1.02, a MATLAB toolbox for optimization over symmetric cones. Optimization Methods and Software, 11-12:625-653, 1999. Special issue on Interior Point Methods (CD supplement with software), http://sedumi.mcmaster.ca/.

[25] E. Tadmor. Filters, mollifiers and the computation of the Gibbs phenomenon. Acta Numerica, (16):305-378, 2007.

[26] E. Tadmor and J. Zou. Three novel edge detection methods for incomplete and noisy spectral data. Journal of Fourier Analysis and Applications, 14(5-6):744-763, 2008.

[27] K. Toh, R. Tütüncü, and M. Todd. SDPT3 4.0 (beta) (software package). Technical report, Department of Mathematics National University of Singapore, September 2006. http://www. math.nus.edu.sg/ mattohkc/sdpt3.html.

[28] A. Viswanathan. Imaging from Fourier Spectral Data: Problems in Discontinuity Detection, Non-harmonic Fourier Reconstruction and Point-spread Function Estimation. PhD thesis, Arizona State University, 2010.

[29] A. Viswanathan, A. Gelb, and D. Cochran. Iterative design of concentration factors for edge detection. Journal of Scientific Computing, to appear, 2011. 
[30] Y. Wang, W. Yin, and Y. Zhang. A fast algorithm for image deblurring with total variation regularization. SIAM Journal on Imaging Sciences, 1(3):248-272, 2008.

[31] R. M. Young. An introduction to nonharmonic Fourier analysis. Academic Press, New York, 2001. 